\title{
Effect of Bifidobacterium bifidum BF-1 on gastric protection and mucin production in an acute gastric injury rat model
}

\author{
A. Gomi, ${ }^{1}$ N. Harima-Mizusawa, H. Shibahara-Sone, M. Kano, K. Miyazaki, and F. Ishikawa \\ Yakult Central Institute for Microbiological Research, Kunitachi, Tokyo, 186-8650, Japan
}

\begin{abstract}
Homeostasis in the stomach environment is maintained by the balance of protective factors such as gastric mucus and aggressive factors such as gastric acid, stress, alcohol, and drugs. An overload of aggressive factors that upsets this balance can induce gastric injury. Fermented milk that contains Bifidobacterium bifidum BF-1 (BF-1), a probiotic strain, and Streptococcus thermophilus YIT 2021 (ST) is known to improve Helicobacter pylori-associated gastritis in humans. Here, we investigated the gastroprotective potential of BF-1 in a rat model of acid-ethanol-induced acute gastric injury to fully elucidate its potential compared with ST. Living BF-1, ST, or vehicle was orally administrated to rats, and acid-ethanol gastric injury was induced 2 $\mathrm{h}$ later. The gastric injury rate was determined and shown to be significantly lower in the BF-1 group than in the vehicle group, which showed a similar level to the ST group. The production of gastric mucin and the expression of several target genes associated with protection and inflammation were examined before and after induction of gastric injury. Interestingly, mucin 5 ac $(m u c 5 a c)$ gene expression in gastric corpus samples and gastric mucin production in stomach samples from the BF-1 group, but not the ST group, were significantly higher than those in the respective samples from the vehicle group. These findings indicate that BF-1 has the potential to provide gastroprotection, alleviating acute gastric injury by enhancing the production of gastric mucin in a rat model.
\end{abstract}

Key words: Bifidobacterium bifidum BF-1, gastroprotective effect, mucin, probiotics

\section{INTRODUCTION}

Homeostasis in the stomach is widely believed to be maintained through balancing protective factors such as gastric mucus, bicarbonates, and gastric mucosal blood flow with aggressive factors such as gastric acid

Received July 17, 2012.

Accepted October 15, 2012.

${ }^{1}$ Corresponding author: atsushi-gomi@yakult.co.jp and pepsins (Boyd and Wormsley, 1985). Distortions of this balance can lead to the development of gastric disorders; collapse of protective factors or the overproduction of aggressive factors, or both, induced by stress, alcohol, or drugs can cause such distortions (Schwarz, 1910; Allen and Garner, 1980; Kauffman, 1981). Recently, colonization of Helicobacter pylori in the stomach has been shown to play an important role in various gastric disorders.

Gastric mucus is the most important protective factor of the gastric mucosa. It is produced by surface mucus cells and gland mucus cells in the stomach and secreted as mucin, which is a glycosylated protein that forms a gel layer on the gastric mucus membrane (Neutra and Forstner, 1987). The mucin production-associated genes mucin 5ac (muc5ac) and mucin 6 (muc6) encode mucin monomers, which are rod-shaped apomucin cores that are posttranslationally glycosylated.

Probiotics are defined by the Food and Agriculture Organization of the United Nations and the World Health Organization (FAO/WHO, 2001) as "live microorganisms, which when administered in adequate amounts confer a health benefit on the host." Lactic acid bacteria and bifidobacteria are the most common types of microbes used as probiotics, and evidence is increasing that some probiotic lactobacilli offer beneficial health effects such as the suppression of harmful bacteria and metabolites in the gut, immune modulation, and mechanisms against anti-bowel disorders, anti-allergy, anti-inflammation, and anti-cancer (Isolauri et al., 2001; Reid and Hammond, 2005; Miyazaki and Matsuzaki, 2008; Cain and Karpa, 2011). Bifidobacteria, which is used as a starter for various fermented milk products and dietary supplements, has proven probiotic effects (Shimakawa et al., 2003; Picard et al., 2005; Yakult Central Institute for Microbiological Research, 2012) and beneficial effects on the stomach. For example, Yamamoto et al. (1994) have reported that the administration of Bifidobacterium bifidum YIT 4007 reduces the severity of peptic ulcers and limits H. pylori infection in the gastric mucosa of affected patients. Nagaoka et al. (1994) found that, in rats, the cell wall polysaccharides of B. bifidum YIT 4007 mitigated the acute gastric injury induced by ethanol 
and acetic acid; moreover, fermented milk that contains Bifidobacterium bifidum BF-1 (BF-1) and Streptococcus thermophilus (ST) improved H. pylori-associated gastritis in a clinical trial, and BF-1 suppressed $H$. pylo$r i$-induced IL-8 secretion of human gastric cells (Miki et al., 2007) by inhibiting expression of $H$. pylori-induced genes and NF- $\kappa \mathrm{B}$ signaling pathway-associated genes (Shirasawa et al., 2010). However, the gastroprotective potential of BF-1 has yet to be fully elucidated in an animal model. Therefore, we used a rat model of acute gastric injury to investigate and compare the protective potential of living BF-1 with that of living ST.

\section{MATERIALS AND METHODS}

\section{Bacteria and Culture}

Bifidobacterium bifidum BF-1 (B. bifidum YIT 10347) and ST (Strep. thermophilus YIT 2021) were obtained from the Culture Collection Research Laboratory of the Yakult Central Institute for Microbiological Research (Tokyo, Japan). Bifidobacterium bifidum BF-1 was cultured in modified international lactobacilli Shirota (mILS) broth (Shimakawa et al., 2003) under anaerobic conditions using an AnaeroPack system (Mitsubishi Gas Chemical Co. Inc., Tokyo, Japan) for $20 \mathrm{~h}$ at $37^{\circ} \mathrm{C}$.

Streptococcus thermophilus was grown in Difco lactobacilli de Man, Rogosa, and Sharpe (MRS) broth (Becton Dickinson Co., Sparks, MD) in static culture for $20 \mathrm{~h}$ at $37^{\circ} \mathrm{C}$. Cells of each bacterial strain were collected by centrifugation at $3,000 \times g$ for $10 \mathrm{~min}$ at $4^{\circ} \mathrm{C}$, washed twice in PBS, and resuspended in cold PBS. Before administration to rats, the number of colonyforming units in individual BF-1 or ST samples was determined by culturing samples on plates containing TOS propionate agar medium (Yakult Pharmaceutical Industry Co. Ltd., Tokyo, Japan) or Difco lactobacilli MRS agar (Becton Dickinson Co.).

\section{Animals}

All rats were maintained and treated in accordance with the guidelines of the Ethical Committee for Animal Experiments of the Yakult Central Institute for Microbiological Research. Male Sprague-Dawley rats [8 wk old, specific-pathogen-free (SPF) grade] were obtained from CLEA Japan Inc. (Tokyo, Japan) and were then housed in cages with wire mesh bottoms to prevent coprophagia. The cages were kept in a room with controlled lighting (lights on 0830 to $2030 \mathrm{~h}$ ), temperature $\left(25 \pm 1^{\circ} \mathrm{C}\right)$, and humidity $(60 \pm 5 \%)$. Rats were given free access to a commercial nonpurified solid diet (MF diet; Oriental Yeast Co. Ltd., Tokyo, Japan) and tap water.

\section{Administration of Bacteria}

After a 7 -d adaptation period, rats $(\mathrm{n}=36)$ weighing between 280 and $320 \mathrm{~g}$ were randomly divided into 3 groups. All rats were fasted for $20 \mathrm{~h}$ before the experiment, but had free access to water. Suspensions containing fresh BF-1 or fresh ST (each $7.5 \times 10^{10} \mathrm{cfu} / \mathrm{mL}$ per rat) or vehicle only (PBS) were orally administered through a stainless tube attached to a $2.5-\mathrm{mL}$ syringe.

\section{Induction and Evaluation of Gastric Injury}

To induce acute gastric injury, an aqueous solution containing $60 \%$ ethanol and $150 \mathrm{~m} M$ hydrochloride (1 $\mathrm{mL} / \mathrm{rat}$ ) was intragastrically instilled $2 \mathrm{~h}$ after administration of the bacterial suspension. For surgical collection of stomach samples, the rats were anesthetized under $\mathrm{CO}_{2} 1 \mathrm{~h}$ after application of the ethanol-acid solution and then immediately killed for collecting samples. After the removal of gastric fluid, each stomach was inflated by injection of $10 \mathrm{~mL}$ of $2 \%$ formalin, and tissues in the inflated stomachs were fixed for $10 \mathrm{~min}$. Thereafter, each stomach was opened along the line of greater curvature, washed in PBS, and spread on a board. To determine the total injury area, the length $(\mathrm{mm})$ and width $(\mathrm{mm})$ of the gastric lesions (areas of red streaks) were measured by an observer that was blinded to the treatment groups; the injury area was expressed as the lesion index. The percentage of injury was calculated using the following equation: lesion index of the test group/lesion index of the vehicle group (control group) $\times 100$. The drug benexate hydrochloride betadex (45 and $90 \mathrm{mg} / \mathrm{rat}$; Shionogi \& Co. Ltd., Osaka, Japan) was used as a positive control.

\section{Analysis of mRNA Expression}

Stomachs that had been obtained surgically were opened along the line of greater curvature and divided into right and left halves. After washing with PBS, tissue samples were collected from the fundic region in the right half of the stomach and immediately submerged in RNA-protective solution for $18 \mathrm{~h}$ at $4^{\circ} \mathrm{C}$. Tissue samples were then homogenized in an acid guanidinium thiocyanate-phenol-chloroform mixture (ISOGEN; Nippon Gene Co. Ltd., Toyama, Japan), and total RNA was extracted from these homogenates. To analyze the expression of 7 genes ( 4 rat genes, 2 bacterial genes, and 1 rat house-keeping gene; Table 1), real-time reversetranscription PCR was conducted using Power SYBR Green PCR master mix and a 7500 real-time PCR system (Applied Biosystems Inc., Foster City, CA). Rat PCR primers were designed with Primer Express software (Applied Biosystems Inc.); primer design was 
Table 1. Target genes and primers used for real-time reverse-transcription PCR

\begin{tabular}{|c|c|c|c|}
\hline Gene name ${ }^{1}$ & Direction $^{2}$ & $5^{\prime}-3^{\prime}$ sequence & Reference \\
\hline muc $5 a c$ & $\mathrm{R}$ & GACTTAGGACCAGCCTTTTG & \\
\hline muc6 & $\mathrm{F}$ & CATCACCTTTGTCTCCTACG & \\
\hline \multirow[t]{2}{*}{$E G F$} & $\mathrm{~F}$ & CCGAGTCCTGTAGTAGTAAG & \\
\hline & $\mathrm{R}$ & TTACTGCTCACGTTGCTGCT & \\
\hline$b F G F$ & $\mathrm{~F}$ & CTGCTGGCTTCTAAGTGTGT & \\
\hline Bifidobacterium bifidum & $\mathrm{R}$ & ACAACCTGGTCCTCAGTGTA & \\
\hline \multirow[t]{2}{*}{ Streptococcus thermophilus } & $\mathrm{F}$ & GTGTGGATTGGTGGCTCTAT & Watanabe et al. (1998) \\
\hline & $\mathrm{R}$ & GACTCATCGTACTCCTGCTT & \\
\hline \multirow{2}{*}{$\beta$-Actin } & $\mathrm{F}$ & GTGTGGATTGGTGGCTCTAT & \\
\hline & $\mathrm{R}$ & GACTCATCGTACTCCTGCTT & \\
\hline
\end{tabular}

${ }^{1} m u c 5 a c=$ mucin 5 ac; $m u c 6=$ mucin $6 ; E G F=$ epidermal growth factor; $b F G F=$ basic fibroblast growth factor.

${ }^{2} \mathrm{~F}=$ forward; $\mathrm{R}=$ reverse.

based on the transcription sequence data of each gene obtained from PubMed (http://www.ncbi.nlm.nih.gov/ pubmed). Measurements from different RNA samples were normalized for RNA quantity relative to an internal control- $\beta$-actin, a house-keeping gene.

\section{Evaluation of Gastric Mucin Production}

The production of gastric mucin was evaluated using previously reported methods (Ishihara and Hotta, 1993). Briefly, gastric fluid containing soluble mucus was collected by washing a gastric fluid fraction from the left half of the stomach samples that had been prepared as described above as with PBS. The remaining left half of the stomach was immersed in $10 \mathrm{~mL}$ of PBS containing 2\% (wt/vol) $N$-acetylcysteine for 5 min at room temperature to collect an adherent mucus gel layer fraction. Both fractions were combined; these mixtures were subjected to centrifugation at $10,000 \times g$ at $4^{\circ} \mathrm{C}$ for $10 \mathrm{~min}$, and the supernatants were collected and defined as the gastric mucin fraction. As an index of gastric mucin production, the sugar content in the gastric mucin fraction was quantified by the phenolsulfuric acid method; glucose was used as a standard for these measurements.

\section{Statistical Analysis}

Data are presented as means \pm standard deviation. Statistical significance was determined using the Steel test or Dunnett test and $P<0.05$ was considered to be statistically significant.

\section{RESULTS}

\section{Effects on Acute Gastric Injury}

Preliminary experiments demonstrated that the oral administration of a positive control, benexate hydro- chloride betadex, significantly inhibited the induction of injury; specifically, the positive control resulted in an approximate injury area that was $30 \%$ that of the vehicle administration group (data not shown). The effects of oral administration of living BF-1 were then examined in this rat model of acute gastric injury. Images of the stomachs of rats administered vehicle or $\mathrm{BF}-1$, and then $2 \mathrm{~h}$ later treated with ethanol and acid to induce acute gastric injury, are shown (Figure 1). The rats given BF-1 exhibited relatively minor acute injury similar to a gastric ulcer in the corpus; these injuries were less severe than those of rats given vehicle. Figure 2 shows the rate of injury area in the rats given vehicle, $\mathrm{BF}-1$, or ST $\left(7.5 \times 10^{10} \mathrm{cfu} / \mathrm{rat}\right)$. The rate of injury area was significantly lower in the BF-1 group $(48.9 \pm 43.2 \%)$ than in the vehicle group (100 $\pm 63.3 \%$ ), which had a similar rate to the ST group $(89.0 \pm 52.4 \%)$. These findings indicate that the oral administration of living BF-1 has a gastroprotective potential that mitigated acute gastric injury in this rat model. Administration of BF-1 $\left(7.5 \times 10^{10} \mathrm{cfu} / \mathrm{rat}\right)$ $4 \mathrm{~h}$ before induction of gastric injury had significant gastroprotective potential, but administration of BF-1 $\left(1.0 \times 10^{10} \mathrm{cfu} / \mathrm{rat}\right) 2 \mathrm{~h}$ before gastric injury did not have significant effect (data not shown).

\section{Effect on Stomach mRNA Expression}

Next, to understand the effects of the administration of living BF-1 on the stomach, we analyzed the expression of several genes (Table 1) in the gastric corpus of rats. Bifidobacterium bifidum gene expression was observed in the BF-1 group $\left(5.6 \times 10^{-2} \pm 1.1 \times\right.$ $0^{-1} / \beta$-actin) and Strep. thermophilus gene expression was observed in the ST group $\left(4.7 \times 10^{-5} \pm 6.6 \times\right.$ $10^{-5} / \beta$-actin), but this bacterial expression was not observed in the other group. A gene associated with 


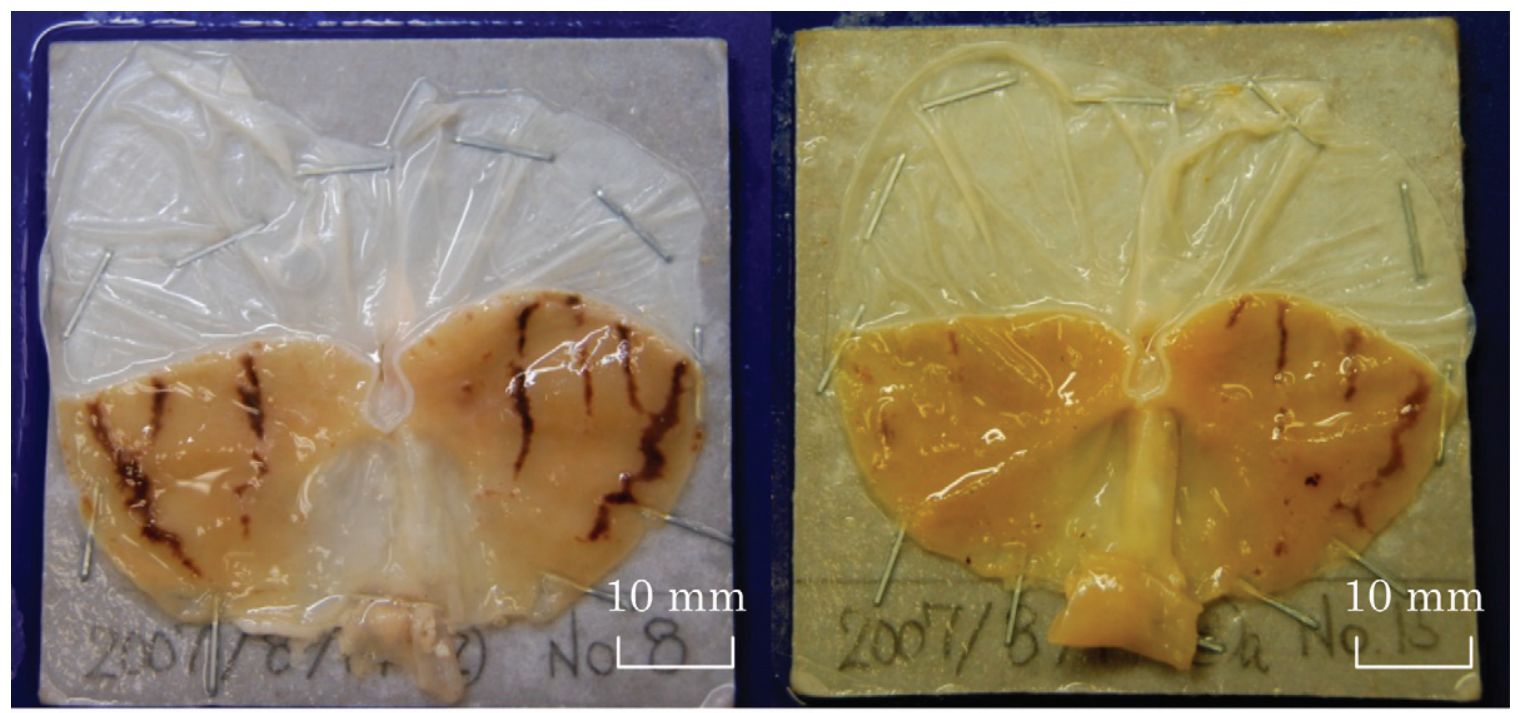

Vehicle

BF-1

Figure 1. Representative images of acute gastric injury in rats. Rats were given vehicle (PBS) or Bifidobacterium bifidum BF-1 (BF-1) and then treated with an aqueous solution of $60 \%$ ethanol with $150 \mathrm{mM}$ hydrochloride $(1 \mathrm{~mL} / \mathrm{rat})$ to induce acute gastric injury.

gastric protection $(m u c 5 a c)$ was expressed in rats that had been treated with BF-1, ST, or vehicle before the induction of acute injury (Figure 3). Expression of the muc5ac mRNA was significantly higher in the BF-1 group $(183.8 \pm 50.6 \%)$ than in the vehicle group (100 $\pm 21.2 \%$ ), but no significant difference existed in $m u$ c5ac mRNA expression between the ST group (122.2 \pm $28.2 \%$ ) and the vehicle group or the BF-1 group. Expression of each of the other 3 genes monitored was not significantly affected by ST or BF-1 treatment (data not shown).

\section{Effect on Gastric Mucin Production}

We also assessed the effect of BF-1 and that of ST on the production of mucin derived from muc5ac expression in the stomach. The sugar content in the gastric mucin fraction was significantly higher in the BF-1 group $(129.74 \pm 32.2 \%)$ than in the vehicle control group (100 $\pm 17.4 \%)$, which had a similar content to that of the ST group $(86.6 \pm 23.2 \%)$. This finding indicates that oral administration of living BF-1 enhances mucin production in the rat stomach.

\section{DISCUSSION}

Reportedly, some probiotics have beneficial effects in the stomach in animal models and clinical trials. In particular, fermented milk containing BF-1 and ST reportedly alleviated $H$. pylori-associated gastritis in hu- mans (Miki et al., 2007). However, the gastroprotective potential of BF-1 in acute gastric injury is unknown. Therefore, this study was designed to examine and compare the protective potential of living BF-1 and ST in an acute gastric injury rat model and to examine the underlying mechanisms.

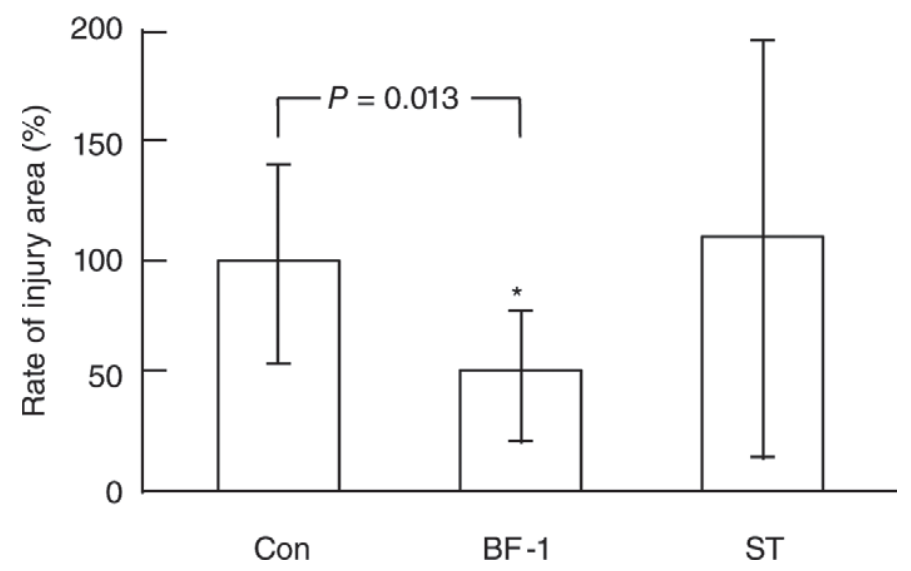

Figure 2. Effect of oral administration of Bifidobacterium bifidum BF-1 (BF-1) or Streptococcus thermophilus (ST) on acute injury in ethanol-acid-treated rats. The administration of each sample and the induction of injury are described in Figure 1. The injury area was measured, and the percentages of injury area were calculated as follows: lesion index of the test group/lesion index of the vehicle (PBS) group $($ control group, Con $) \times 100$. Rats that had exogenous materials in the stomach samples were excluded from the analysis. Data are expressed as means \pm SD. Con group: $\mathrm{n}=12$; BF- 1 group: $\mathrm{n}=11$; ST group: $\mathrm{n}$ $=12$. $P$-values were calculated using the Steel test. ${ }^{*} P<0.05$. 


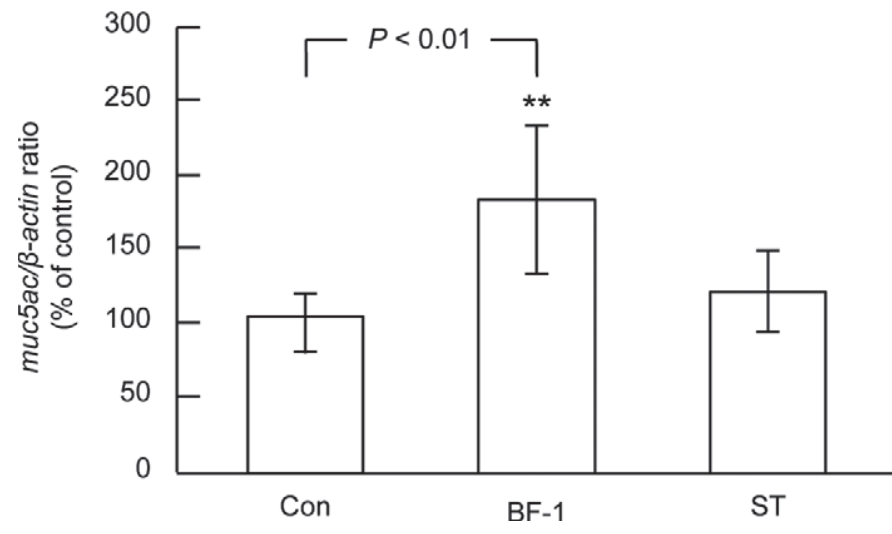

Figure 3. Effect of oral administration of Bifidobacterium bifidum BF-1 (BF-1) or Streptococcus thermophilus (ST) on mucin 5ac (muc5ac) gene expression in the stomach of rats. The expression of target genes shown in Table 1 was analyzed by real-time reverse-transcription PCR. The ratio of muc5ac expression to $\beta$-actin expression was determined for each group and expressed as a percentage relative to the vehicle (PBS) group (Con). Rats that had exogenous materials in the stomach samples were excluded from the analysis. Data are expressed as means \pm SD. Con group: $\mathrm{n}=12$; BF- 1 group: $\mathrm{n}=10$; ST group: $\mathrm{n}=10 . P$-values were calculated using the Dunnett test. ${ }^{*} P P<0.01$.

Oral administration of living BF-1 showed gastroprotective potential that mitigated acute gastric injury in this rat model, but administration of living ST did not (Figure 2). These findings support the concept that BF-1 is one, and possibly the only, component of fermented milk that is responsible for the mitigation of $H$. pylori-associated gastritis in humans. However, the gastroprotective potential of BF-1 at a dose of 7.5 $\times 10^{10} \mathrm{cfu} / \mathrm{rat}$ was such that the injury area of the BF-1 group was approximately $50 \%$ that of the vehicle group, and this protective potential was milder than that of benexate hydrochloride betadex at a dose of $45 \mathrm{mg} / \mathrm{rat}$; benexate hydrochloride betadex resulted in an injury area that was approximately $30 \%$ that of the vehicle group.

Our findings also demonstrated that within $2 \mathrm{~h}$ after administration of living BF-1, but not living ST, mucin production was enhanced at both the mRNA level and the sugar content level in the stomach of rats (Figures 3 and 4). Reportedly, the cell wall polysaccharide of B. bifidum strain YIT 4007 has a similar gastroprotective potential in acetic acid and ethanol-treated rats; in these rats, it stimulates the production of epidermal growth factor and basic fibroblast growth factor in gastric tissue within $4 \mathrm{~h}$ after administration (Nagaoka et al., 1994). However, our study indicated that mRNA expression of epidermal growth factor and basic fibroblast growth factor was unchanged in the gastric corpus of the stomach $2 \mathrm{~h}$ after the administration of BF-1.

Mucin is a glycoprotein that forms the mucus gel layer that adheres to and protects the epithelial tis-

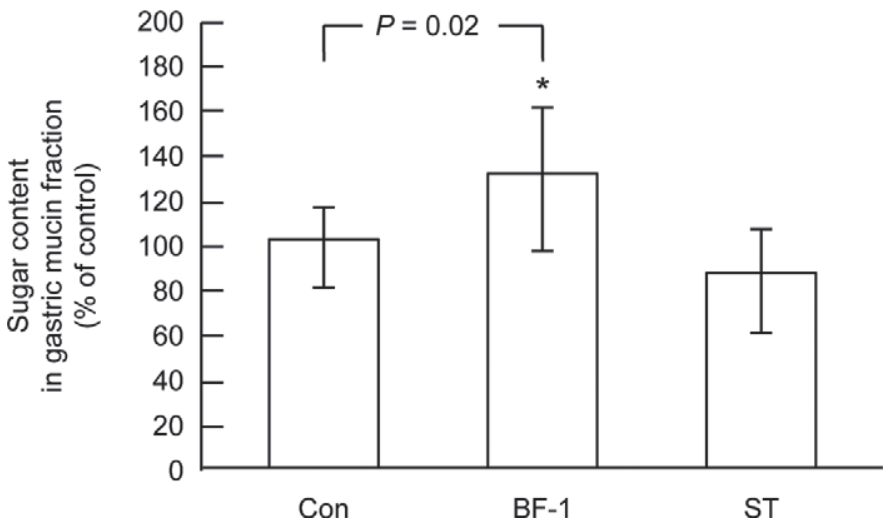

Figure 4. Effect of oral administration of Bifidobacterium bifidum BF-1 (BF-1) or Streptococcus thermophilus (ST) on mucin production in rat stomach. Sugar content in the gastric mucin fraction prepared from rat stomachs was measured to evaluate mucin production in each group, and the data from the BF-1 and the ST groups were expressed as a percentage relative to the vehicle (PBS) group (Con). Rats that had exogenous materials in the stomach samples were excluded from the analysis. Data are expressed as means \pm SD. Con group: $\mathrm{n}=12$; BF-1 group: $\mathrm{n}=8$; ST group: $\mathrm{n}=10$. $P$-values were calculated using the Dunnett test. ${ }^{*} P<0.05$.

sue of the gastrointestinal tract. Helicobacter pylori infection reportedly causes the mucus gel layer in the stomach to become thinner (Newton, 2000); similarly, nonsteroidal antiinflammatory drugs decrease the magnitude of the mucus-bicarbonate barrier, disrupt the epithelial cell layer, reduce the surface hydrophobicity of epithelial cells, and diminish mucosal blood flow (Shorrock and Rees, 1989). Our findings indicate that the enhancement of mucin production following the oral administration of living BF-1 is an early response that plays an important role in protection against acute gastric injury in rats. In addition, BF-1 could have a gastroprotective function against the decrease of the mucus gel layer induced by H. pylori, nonsteroidal antiinflammatory drugs, or other factors in humans.

It is known that diluted acetic acid such as vinegar acts as a mild irritant to the stomach, resulting in adaptive cytoprotection with an increase of gastric mucosal blood flow. Kawauchi et al. (2000) have reported that 0.3 to $1.0 \%$ acetic acid, but not $0.1 \%$ acetic acid, given acutely also reduce the severity of $\mathrm{HCl} /$ ethanol-induced gastric lesions. In our study, the concentration of acetic acid in BF-1 and ST cell suspension was $0.1 \%$, and lactic acid in the suspension was less than the detection level $(0.03 \%)$. This suggests that $0.1 \%$ acetic acid in the cell suspension could not influence the suppression of $\mathrm{HCl}$ /ethanol-induced gastric lesions in our study. Nagaoka et al. (1994) have reported that the cell wall polysaccharide of B. bifidum YIT 4007 had gastroprotective potential in acetic acid- and ethanol-treated rats. Therefore, it is suggested that mechanisms other 
than those of lactic acid and acetic acid are associated with the gastroprotective potential of BF-1.

Based on in vitro assays, BF-1 adhered more tightly to gastric epithelial cells and to gastric mucus than did other strains of bifidobacteria and lactobacilli [A. Gomi, H. Shibahara-Sone, T. Iino (Yakult Central Institute for Microbiological Research, Tokyo, Japan), Y. Shimakawa (Yakult Central Institute for Microbiological Research), K. Miyazaki, and F. Ishikawa, unpublished data], indicating some interaction with the BF-1 on the gastric mucosa and gastric epithelial cells. Interestingly, we found that the BF-1 cells were 1,000 times more common than the ST cells in the stomach samples examined in this study. Moreover, BF-1 could tolerate gastric acid well, surviving for at least $4 \mathrm{~h}$ in the stomach of rats after oral administration (unpublished data). These characteristics may explain the observation that BF-1 enhances mucin production and offers subsequent gastroprotection. Alternatively, the possibility exists that BF-1 in the gut plays multiple roles in protection against acute gastric injury. More comprehensive studies are necessary to clarify the mechanism of the gastroprotective potential of BF-1.

\section{CONCLUSIONS}

We propose that BF-1 is a useful probiotic strain with the potential to confer gastroprotection and alleviate acute gastric injury by enhancing gastric mucin production. Further clinical trials of BF-1 treatments are necessary to determine whether such treatments could benefit human patients with gastric problems.

\section{ACKNOWLEDGMENTS}

We express our gratitude to Masato Nagaoka (Yakult Central Institute for Microbiological Research, Tokyo, Japan) for his valuable advice. We also thank Toru Iino, Yasuhisa Shimakawa, Satoshi Matsubara, Norie Onodera-Masuoka, and Norihiro Kubota of our laboratory (Yakult Central Institute for Microbiological Research) for technical help and discussions.

\section{REFERENCES}

Allen, A., and A. Garner. 1980. Mucus and bicarbonate secretion in the stomach and their possible role in mucosal protection. Gut 21:249-262.

Boyd, E. J. S., and K. G. Wormsley. 1985. Etiology and pathology of peptic ulcer. Pages 1013-1059 in Bockus Gastroenterology. Vol. 2. J. E. Berk, ed. W. B. Sanders Co, Philadelphia, PA.

Cain, A. M., and K. D. Karpa. 2011. Clinical utility of probiotics in inflammatory bowel disease. Altern. Ther. Health Med. 17:72-79.
FAO/WHO (Food and Agriculture Organization of the United Nations/World Health Organization). 2001. Health and nutritional properties of probiotics in food including powder milk with live lactic acid bacteria. Report of a joint FAO/WHO expert consultation. FAO, Rome, Italy and WHO, Geneva, Switzerland.

Ishihara, K., and K. Hotta. 1993. Comparison of the mucus glycoproteins present in the different layers of rat gastric mucosa. Comp. Biochem. Physiol. B 104:315-319.

Isolauri, E., Y. Sütas, P. Kankaanpää, H. Arvilommi, and S. Salminen. 2001. Probiotics: Effects on immunity. Am. J. Clin. Nutr. 73:444S-450S.

Kauffman, G. L. 1981. Gastric mucus and bicarbonate secretion in relation to mucosal protection. J. Clin. Gastroenterol. 3:45-50.

Kawauchi, S., H. Mimaki, H. Kitazaki, and K. Takeuchi. 2000. Effect of dilute acetic acid on rat gastric mucosa following acute and chronic administration. Jpn. Pharmacol. Therapeut. 28:473-480.

Matsuki, T., K. Watanabe, J. Fujimoto, Y. Kado, T. Takada, K. Matsumoto, and R. Tanaka. 2004. Quantitative PCR with 16S rRNA-gene-targeted species-specific primers for analysis of human intestinal bifidobacteria. Appl. Environ. Microbiol. 70:167-173.

Miki, K., Y. Urita, F. Ishikawa, T. Iino, H. Shibahara-Sone, R. Akahoshi, S. Mizusawa, A. Nose, D. Nozaki, K. Hirano, C. Nonaka, and T. Yokokura. 2007. Effect of Bifidobacterium bifidum fermented milk on Helicobacter pylori and serum pepsinogen levels in humans. J. Dairy Sci. 90:2630-2640.

Miyazaki, K., and T. Matsuzaki. 2008. Health properties of milk fermented with Lactobacillus casei strain Shirota (LcS). Pages 165172 in Handbook of Fermented Functional Foods. 2nd ed. R. F. Edward, ed. CRC Press, Boca Raton, FL.

Nagaoka, M., S. Hashimoto, T. Watanabe, T. Yokokura, and Y. Mori. 1994. Anti-ulcer effects of lactic acid bacteria and their cell wall polysaccharides. Biol. Pharm. Bull. 17:1012-1017.

Neutra, M. R., and J. E. Forstner. 1987. Gastrointestinal mucus: Synthesis, secretion, and function. Pages 975-1009 in Physiology of the Gastrointestinal Tract. Vol. 2. L. R. Johnson, ed. Raven Press, New York, NY.

Newton, J. L. 2000. The adherent gastric antral and duodenal mucus gel layer thins with advancing age in subjects infected with Helicobacter pylori. Gerontology 46:153-157.

Picard, C., J. Fioramonti, A. Francois, T. Robinson, F. Neant, and C. Matuchansky. 2005. Bifidobacteria as probiotic agents-Physiological effects and clinical benefits. Aliment. Pharmacol. Ther. 22:495-512.

Reid, G., and J. A. Hammond. 2005. Probiotics. Some evidence of their effectiveness. Can. Fam. Physician 51:1487-1493.

Schwarz, K. 1910. Über penetrierende Magen- und Jejunalgeschwure. Beitr. Klin. Chir. 67:96-128.

Shimakawa, Y., S. Matsubara, N. Yuki, M. Ikeda, and F. Ishikawa. 2003. Evaluation of Bifidobacterium breve strain Yakult-fermented soymilk as a probiotic food. Int. J. Food Microbiol. 81:131-136.

Shirasawa, Y., H. Shibahara-Sone, T. Iino, and F. Ishikawa. 2010. Bifidobacterium bifidum BF-1 suppresses Helicobacter pylori-induced genes in human epithelial cells. J. Dairy Sci. 93:4526-4534.

Shorrock, C. J., and W. D. W. Rees. 1989. Mechanisms of gastric damage by non-steroidal anti-inflammatory drugs. Scand. J. Rheumatol. Suppl. 78:5-11, discussion 30-32.

Watanabe, K., T. Miyake, T. Matsuki, and H. Oyaizu. 1998. The use of 16SrRNA gene sequences to identify Lactobacillus species in fermented daily products. Pages 129-154 in Molecular Ecology of the Intestinal Flora. T. Mitsuoka, ed. Japan Scientific Societies Press, Tokyo, Japan.

Yakult Central Institute for Microbiological Research. 2012. Advances in the Science of Bifidobacterium Yakult Strains - Intestinal Microbiota and Human Health. Yakult Honsha Co. Ltd., Tokyo, Japan. Yamamoto, Y., S. Sakurabayashi, K. Abe, S. Sezai, M. Hirano, and H. Oka. 1994. Anti-ulcer effect of Bifidobacterium bifidum YIT 4007. Jpn. Pharmacol. Therapeut. 22:4743-4746. (In Japanese.) 